\title{
Learning Programming with Educational Robotics: Towards an Integrated Approach
}

\author{
Marios Xenos ${ }^{(\bowtie)}$, Nikoleta Yiannoutsou, Marianthi Grizioti, Chronis Kynigos, \\ and Sofia Nikitopoulou
}

Educational Technology Lab, Department of Pedagogy, School of Philosophy, National and Kapodistrian University of Athens, Athens, Greece

\{mariosxenos, nyiannoutsou, mgriziot,

kynigos, sophieniki\} @ppp.uoa.gr

\begin{abstract}
Despite the fact that it has been a few years since robotics entered the school and offered new learning opportunities, educational robotics usually is offered in the context of extra-curricular activity (e.g. a "club") which addresses a limited number of students and participation is based on student personal interest. In this paper we explore the potential of ER when it is integrated in the typical school curriculum. In the study we report here, we integrated ER in the computer science curriculum and all students of a $9^{\text {th }}$ grade class engaged with robotics activities. The rationale underlying the study is that robotics can be used as a medium to motivate students in engaging with programming and support them to negotiate real life problems. Analysis of the data collected, indicate that ER when integrated with the computer science curriculum, can create a rich learning environment where programming is contextualized and students are highly motivated to engage and negotiate important STEM concepts.
\end{abstract}

Keywords: Constructionism $\cdot$ Programming $\cdot$ Educational robotics

\section{Introduction}

It has been over three decades since Papert and his Constructionism Theory expressed the important role that computation technology plays in overcoming mind difficulties when students construct their own knowledge [1]. A few years later computers entered everyday life and became a necessity for everyone. Later on, we started talking about digital fluency [2] and presented the potential uses of robotics in education [3]. Nowadays, the World Robot Olympiad has been enriched with many different categories in order to motivate the participation of students of all education levels [4] indicating the role of Educational Robotics (hereafter ER) in the school.

In many countries the ER program is offered as a separate domain or in special school "clubs" where a limited number of students participate. In this paper we present a study which takes place in real classroom settings and focuses on integrating robotics in the curriculum of Computer Science (hereafter CS). The study design does not try to replace a 
part of CS curriculum with another approach using ER but it offers an additional environment to enrich and support CS concepts when all students of a class are engaged. The aim of the study is to identify outcomes, difficulties and advantages in this integrated approach.

\section{The Study}

The study was conducted at a Junior High School in Attiki (Greece) in the context of ER4STEM - a three-year project, funded by the European Commission -which focuses on the development of an integrated framework of the different European approaches in using Robotics in Education. Six 9th grade classes, of 65 students in total participated in the study, which lasted 6 school hours distributed in 6 weeks time. The participants worked with robotics for the first time but they had short previous experience in programming with blocks using Scratch. The research method we used is that of DesignBased Research which includes the design of a pedagogical intervention and its evaluation in real classroom settings with the aim to refine the initial pedagogical design [5]. Design - Based Research focuses mainly on the collection and analysis of qualitative data as the objective is to identify the main characteristics and the different facets of the designed intervention when implemented with students.

\subsection{The Task}

As we mentioned earlier in this paper the task was mainly oriented towards programming but it did not exclude an engineering aspect. Specifically, the study included a task, which consisted of two main activities, an engineering activity and a programming activity.

The engineering activity was the first activity of the task in the context of which students were expected to adjust an ultrasonic sensor in a preassembled vehicle. The challenge for the students was to keep the construction robust and reliable so that the robot could use the sensor for indentifying obstacles. To this end, students were expected to identify the robot shape, the motion direction and the sensor's best position for obstacle identification. An additional requirement was, for other parts of the robot to not interfere with the sensor and the sensor should not intercept the functioning of the robot.

The programming activity was about defining the behavior of the robot so as to be able to identify obstacles while moving. In this activity, the teacher gave to the students a predefined simple program which allowed the robot to detect obstacles. Students were asked to adjust the program so that the robot would detect an obstacle at a specific distance and react avoiding the obstacle with a predefined movement (i.e. performing a $\mathrm{U}$ turn and stopping). The programming concepts involved in this activity include sequential command execution (sequence structure) and a real world calculation problem, as students should test and explore if their robot detected with accuracy the obstacle and if this behavior was reliable. Here, the command execution time affects the next robot action, a problem which was expected to be identified and rectified by the students. Regarding the robot reaction, students were asked to move the robot by 4 floor plates, which required relating the program (commands, parameters and values) with 
the robot's real world movement. The final phase of this activity involved students extending the program so that the vehicle could act more realistically by avoiding efficiently any obstacle encountered. In all phases, teacher had designed some additional sub-tasks to engage students that would come quickly to a solution and so have free time (e.g. program improvement, robot fine-tuning).

\subsection{Robotic Kit}

In the study, students used Lego NXT (one robotic kit per group) for the engineering activity and Lego programming language for the programming activity.

\subsection{Data Collection}

During the study, the data collected involved screen capturing during the programming activity and researcher observation notes after the end of each session. The teacher acted also as researcher. At the beginning and at the end of the study, students filled in questionnaires where they expressed their opinions about the actual activity and about robotics, mathematics and science, in general. In the middle of the study, each group prepared a self - evaluation document and shared it with other groups. Finally after the workshops, the teacher - researcher interviewed selected groups of students.

\section{Results}

The analysis of the collected data provides us with strong evidence regarding the acceptance of the new field of robotics and the level of student engagement in the educational process. It also gives some indications about the knowledge and the experience students earned. Next we present some indicative data from student learning activity which shows their active engagement with programming and engineering concepts.

\subsection{The Human Body Analogy: Experimenting with the Sensors}

During the first activity, when students tried to assemble the sensor, the identification of the best sensor position was not a difficult task as students considered sensor as it was "eyes" of a human body. A couple of groups believed that the "eyes" (sensor) should be positioned with accuracy lined up with robot body in order to work properly. The following extract is indicative:

St1: Let's put it (i.e. the sensor) exactly here (they point at the front of the vehicle)

St2: But there are no holes here (available sockets)

St1: If we put it in the back? Will it work? Or it will be head-butt?

St3: Let's do what we can for now (i.e. put in the back) and will see...

St2: Should we ask the teacher?

St1: Ask what?

St2: If it is important where we put... 
In the extract we presented above we can identify two important aspects. One is the use of the analogy of the human body directing students to place the sensor in the front of the vehicle. The other interesting aspect involves the learning process taking place during student experimentation with the sensor. As we mentioned earlier, students formulated their initial hypothesis about the position of the sensor based on the analogy of the human body. However students could not test this hypothesis as when they observed the actual structure of the robot they realized that they could not find a slot for placing the sensor. When encountered with this impasse they followed another direction for their hypothesis formulation by focusing on the affordances of the vehicle (they identified a socket in the middle of the vehicle). As this option sounded counter-intuitive one student suggested to experiment by testing this option ("let's see what we can do") whereas the other suggested to seek confirmation from the teacher (Fig. 1).

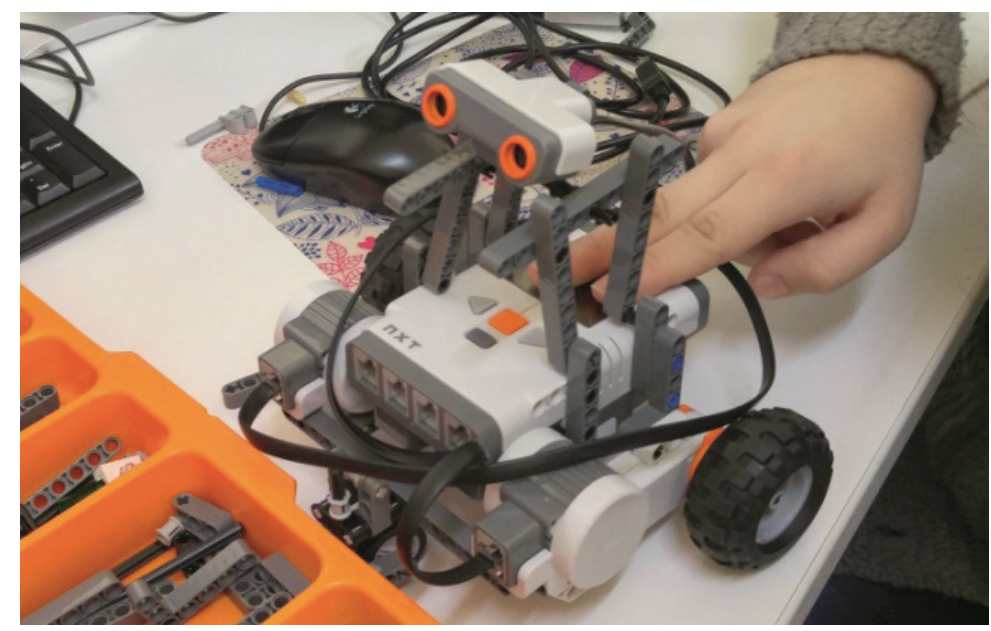

Fig. 1. Students trying to position the sensor

From this simple example the conclusions we can draw are the following: a simple engineering problem can offer rich learning opportunities as students can draw from their experience to make conjectures, then they need to analyze and observe the affordances of the robot to refine these conjectures. Another important aspect in this process is that the physical construction (i.e. placement of the sensor) is tightly linked to the behavior of the robot (if it can detect obstacles or not).

\subsection{Facing the Limitations of Sequential Programming}

During the programming activity students were engaged with the reliable detection of an obstacle. Students for the first time were presented with a tangible and visible result of a sequential command execution in real world: the robot could not check the sensor input until it finishes the previous action. The robot-programming environment provides ready-made solutions for this issue but the study was designed so as to not present them in order for the students to indentify the situation and give their own solutions.

Only 5 out of the 22 groups indentified the problem and suggested a solution. The rest groups attributed the problem to the functioning of the sensor or to its positioning. 
An interesting observation is that most of the students that provided a successful explanation were not good in programming before the study.

St1: Sir, (i.e. teacher) it is doing OK

T: What?

St1: It detects the obstacle

T: At $20 \mathrm{~cm}$ distance? ( $20 \mathrm{~cm}$ was the given distance)

St2: Yes Sir, didn't you see it?

St1: Not exactly at $20 \mathrm{~cm}$. A little shorter

$\mathrm{T}$ : Is it always the same distance?

St1: Hmmm, every time is less. Maybe we could increase the distance (i.e. at the program)?

St2: The program is fine. This (points to the sensor) is the problem

From the student discussion in the extract above it appears that when students discuss with the teacher they purposefully focus on the result of the behavior (i.e. detecting the obstacle) and not on the conditions of this detection (in $20 \mathrm{~cm}$ ). This becomes evident when the teacher asks clarifications. At this point students seem to have identified the problem: i.e. "the distance is a bit shorter" and "every time the distance is less". From this point we see that students differentiate suggesting two directions for repairing the problem: one refers to the program suggesting to increase the given distance which would repair the first part of the identified problem (the distance covered is less than $20 \mathrm{~cm}$ ) but not the second one (every time is less). The other student considers that the problem is related with the sensor and not with the program (Fig. 2).

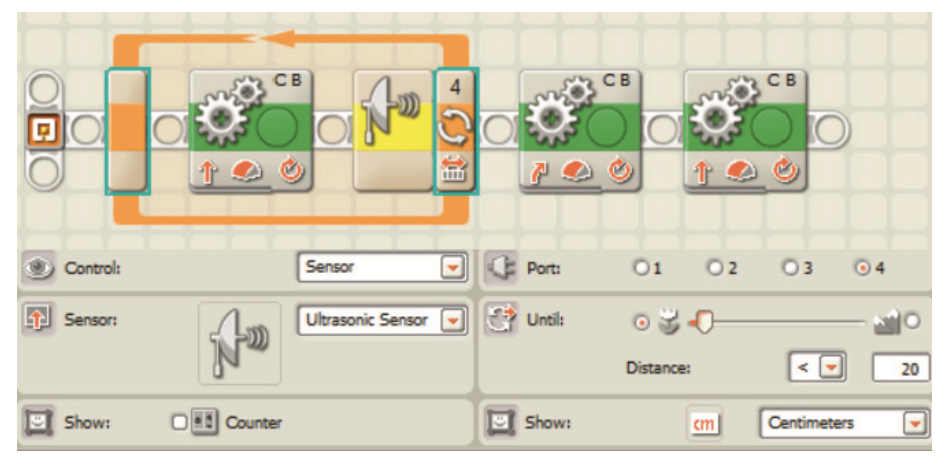

Fig. 2. The result of programming the sensor

Although students in the above extract did not solve the problem at this point, it appears that the structure of the activity and the use of robotics created a learning environment where a) the limitations of sequential programming were clearly demonstrated b) students observing robot behavior could identify in a structured discussion with the teacher the actual limitations of the robot's behavior and c) students could follow different clues in order to pursue solutions to the problem (i.e. the sensor or the program). 


\subsection{Translating Real World Distances into Programming Values}

The next part of the programming activity involved the accurate movement of the robot on 4 floor plates. The strategy that all groups followed was calculations via experimentation. Some groups tried random program values for the whole movement (4 plates). Other groups performed continuous tests on the floor for one plate and then they adjusted their calculation for the 4 plates. None of the groups tried to calculate the distance covered by the robot in relation to the wheel rotation. Even after strong teacher prompting for a computational solution, only few students managed to relate the wheel circumference with the travelling distance.

\subsection{From Sequential Programming to Loops}

The last problem that students had to solve is rather the most interesting in respect to programming and robotics. The obstacle identification should be continuous in order to build a "smart" vehicle. The verbalization of the problem by the teacher helped students to identify the general direction for solution seeking, because when teacher used phrases like "it (the robot) has to do it continuously", "unlimited" "forever", soon students came to the solution of using the "loop structure". However, not all groups put the loop block at the correct position in the program. Some groups put the loop after the obstacle recognition thinking that robot has to find an obstacle and then search for the next one (Fig. 3). Other groups put the loop in the correct position but included only some commands (blocks) and not all. Some other groups put an "empty" loop after initial program thinking that robot can identify the correct use automatically (Fig. 4). Students had none or limited knowledge of nested structures, so faulty usage of loop was not a surprise. This problem was posed in order to engage students with more advanced programming structures and to showcase mainly the realistic robotic aspect giving an almost human behavior to the robot.

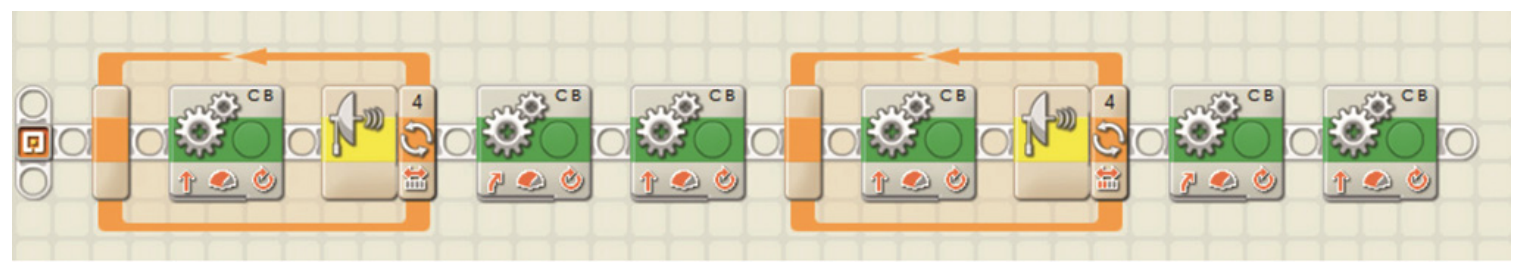

Fig. 3. Incorrect use of the second loop: new loop and new commands after the initial one

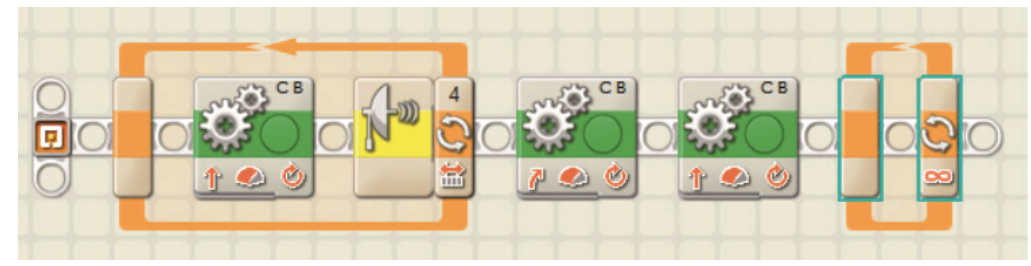

Fig. 4. Incorrect use of the second loop: putting the loop after the initial program 


\subsection{Student Views About Robotics}

Important data was taken by the answers that students gave to anonymous questionnaire after the study. In the evaluation of the task, $90 \%$ of students rated the interest of the activity with 4 or 5 out of 5 . One student gave negative rate and wrote that "it was funny but I was not interested in it". More elaborate information about the participant's opinions regarding the task is found in open questions. Specifically, at the question "What have you learned about yourself?" only 6 students did not answer or answer negatively. The rest of the students gave positive and in many cases long answers:

"I learned to program a robot which can be very useful for me later in my career or in my life generally"

"Through robotics I understood how things work in everyday life and I realized that robotics can make us smarter"

"Eventually, I realized that I do like problem solving projects such as building a robot and make it functional"

“Although I don't like mathematics, I managed to use it and I realized that if you work hard you will succeed"

At the question "What have you learned about working with robots?" only 4 (out of 65 ) students did not answer. The rest of students gave positive answers focusing on the learning results or on the difficulties of the activities:

"It is you that can program them whatever you like and they cannot step away from your orders"

"Although it is sometimes difficult in the end the result is great"

"Working with robots is very interesting. It teaches you how to think in alternative ways in order to achieve your goals"

"I realized that nothing is impossible and with a little hard work I can construct and program a robot"

The overall student interest in robotics and also the importance of robotics in everyday life is designated at the general evaluation answers:

"It was a very interesting course in the field of Computer Science which helped us to understand how things work and how robots are tightly connected with mathematics and physics"

"At the beginning, I wasn't interested at all. But afterwards I understood how important robots are and how important is to collaborate well"

"I had a great time and it was the first time that I programmed. This workshop helped me understand is the importance of mathematics, which I didn't find important before".

\section{Concluding Remarks}

The data presented earlier showed that an integrated approach of robotics in the curriculum of computer science can offer a learning environment which is engaging for all students and provides opportunities for negotiation and elaboration of important programming concepts. Furthermore, students of both sexes even if they believed that robotics and programming does not interest them, they managed to have a fruitful collaboration and engaged actively with the learning activities. Most of the participants realized the different domains integrated in robotics and they stated that they learned a lot of different things. For some students, robotics was a motive to get involved with 
programming and realize an authentic aspect of it. Because of prior knowledge and study design itself, we cannot argue that students learned or understood programming structures even if they think so. However, they had the chance to practice and test CS concepts, explore the results and indentify the connection between programming and real world. On the other hand, it seems that students have a difficulty to transfer prior programming knowledge from one programming environment (Scratch) to another (LEGO), so maybe we have to rethink and reconsider about the tools that typical curriculum uses. A possible side benefit is that all participants stressed the role of collaboration and the importance of team spirit for goal achievement. Our analysis of questionnaires, student constructions and researcher reflection notes, indicates that integration of educational robotics in school curriculum may contribute in:

- Engaging all students in programming especially when they believe it is a boring and difficult subject.

- Presenting career opportunities in the field of computer science and technology

- Demystifying domains that by default are considered as difficult (robotics, mathematics)

- Helping children to understand how things work in everyday life

- Helping students in developing problem solving skills

In conclusion, integrating Educational Robotics into the typical school curriculum seems to be beneficial for a number of reasons. Well-designed activities can result to a rich outcome not only in a specific domain but in many aspects of learning process. It is critical to examine and perform more focused analysis in each aspect of this learning process, in order to prepare a new enriched curriculum.

Acknowledgments. The research reported here has received funding from H2020 project Educational Robotics for STEM: ER4STEM (No. 665972).

\section{References}

Papert, S.: Mindstorms: Children, Computers, and Powerful Ideas. Basic Books Inc, New York (1980)

Resnick, M.: Rethinking learning in the digital age. In: Kirkman, G. (ed.) The Global Information Technology Report: Readiness for the Networked World. Oxford University Press (2002)

Malec, J.: Some thoughts on robotics for education. In: AAAI Spring Symposium on Robotics and Education (2001)

World Robot Olympiad. http://www.wroboto.org

Cobb, P., Confrey, J., diSessa, A., Lehrer, P., Schauble, L.: Design experiments in educational research. Educ. Researcher 32(1), 9-13 (2003) 